\title{
Comparative evaluation of two olive fruit fly parasitoids under varying abiotic conditions
}

\author{
Xin-geng Wang • Marshall W. Johnson • \\ Victoria Y. Yokoyama • Charles H. Pickett • \\ Kent M. Daane
}

Received: 13 June 2010/Accepted: 28 October 2010/Published online: 12 November 2010

(C) The Author(s) 2010. This article is published with open access at Springerlink.com

\begin{abstract}
Psyttalia lounsburyi (Silvestri) and $P$. humilis (Silvestri) (Hymenoptera: Braconidae) were evaluated in California for their potential to control the invasive olive fruit fly, Bactrocera oleae (Rossi) (Diptera: Tephritidae). Psyttalia lounsburyi is a specialist on $B$. oleae while $P$. humilis also attacks other tephritid species. Field cage trials, conducted from 2006 to 2009, were used to compare P. lounsburyi and two populations of $P$. humilis (Kenya and
\end{abstract}

Handling Editor: Dirk Babendreier

X. Wang · K. M. Daane ( $\square)$

Department of Environmental Science,

Policy and Management, University of California,

Berkeley, CA 94720, USA

e-mail: daane@uckac.edu

X. Wang

e-mail: xgwang@uckac.edu

M. W. Johnson

Department of Entomology, University of California,

Riverside, CA 92521, USA

e-mail: mjohnson@uckac.edu

V. Y. Yokoyama

USDA-ARS, San Joaquin Valley Agricultural Sciences

Center, Parlier, CA 93648, USA

e-mail: Victoria.yokoyama@ars.usda.gov

C. H. Pickett

Biological Control Unit, California Department of Food and Agriculture, Sacramento, CA 95832, USA

e-mail: cpickett@cdfa.ca.gov
Namibia) in California's interior valley and coastal regions. Both parasitoid species reproduced on B. oleae in all trials. Under similar abiotic conditions, offspring production per female was higher in $P$. humilis than in $P$. lounsburyi, suggesting that host specificity by $P$. lounsburyi does not confer a higher efficiency on $B$. oleae in cultivated olives. Two abiotic factors were shown to impact parasitoid efficiency. First, adult parasitoid survival was poor during periods of high summer temperatures, common to the olive production areas in California's interior valleys. Second, parasitism levels were lower on B. oleae larvae feeding in larger Ascolano $c v$. fruit than in smaller Manzanillo $c v$. fruit. Results are discussed relative to biological control of B. oleae in commercial olives and the usefulness of natural enemies specialized to attack fruit flies in wild olives compared with the larger cultivated olive fruit.

Keywords Bactrocera oleae - Psyttalia concolor . Psyttalia humilis · Psyttalia lounsburyi - Classical biological control · Field-cage evaluation . Host specificity $\cdot$ Climatic adaptability

\section{Introduction}

The olive fruit fly, Bactrocera oleae (Rossi) (Diptera: Tephritidae), was discovered in California in 1998, rapidly spread throughout all olive growing regions, and now threatens the state's table and oil olive 
industries (Rice et al. 2003; Zalom et al. 2009). High density B. oleae populations occur in California's coastal olive regions while population density and crop infestation is generally lower in the interior valleys (Burrack et al. 2008; Yokoyama et al. 2006) where high summer temperatures may limit population growth (Johnson et al. 2011; Wang et al. 2009a). Studies of indigenous natural enemies in California found that B. oleae larvae are attacked by Pteromalus sp. nr. myopitae (Hymenoptera: Pteromalidae) (Kapaun et al. 2010) and B. oleae pupae are attacked by foraging ants in the ground liter (Orsini et al. 2007). These natural enemies do not adequately suppress B. oleae populations in commercial olives. Moreover, abundant landscape olive trees that are never treated with insecticides serve as reservoirs for the fly's reinvasion into treated commercial orchards (Collier and van Steenwyk 2003). The importation of more specialized B. oleae parasitoids may help suppress fly populations in these situations and thereby lessen levels of fruit damage in commercial orchards (Daane and Johnson 2010).

The California importation program for $B$. oleae natural enemies was initiated in 2003, and surveys have since screened natural enemies of $B$. oleae from the Republic of South Africa, Namibia, Kenya, La Réunion, Canary Islands, Morocco, Pakistan, India, and China (Daane et al. 2011). The wide geographic search for natural enemies was beneficial because B. oleae has distinguishable populations in Africa, Mediterranean Europe, and Pakistan (Augustinos et al. 2002; Nardi et al. 2005), with populations in California most similar to those in the eastern Mediterranean regions (Zygouridis et al. 2009). Collections of natural enemies were most productive from wild olive fruit (Olea europaea ssp. cuspidata) in sub-Saharan Africa (Daane et al. 2011), which is the likely origin of $B$. oleae in cultivated olives (Nardi et al. 2005). From these collections, parasitoids sent to the University of California Berkeley Quarantine Facility, and evaluated for possible field release, included Psyttalia lounsburyi (Silvestri) (Daane et al. 2008), Psyttalia concolor (Szépligeti) (Sime et al. 2006a), Bracon celer Szépligeti (Nadel et al. 2009), and Psyttalia ponerophaga (Silvestri) (Sime et al. 2007) (all Hymenoptera: Braconidae). Utetes africanus (Silvestri) was imported but proved difficult to rear and, therefore, quarantine studies were not completed. Additonally, other braconid parasiotids reared from other fruit fly species were also evaluated, these included Fopius arisanus (Sonan) (Sime et al. 2008), Dichasmimorpha kraussii (Fullaway) and D. longicaudata (Ashmead) (Sime et al. 2006b). These more generalist parasitoids have been used widely in tephritid fruit fly biological control programs.

Psyttalia lounsburyi is a specialist B. oleae parasitoid, as indicated by earlier field surveys in which $P$. lounsburyi was only reared from $B$. oleae (Neuenschwander 1982; Silvestri 1914; Wharton et al. 2000) and results from non-target studies in quarantine (Daane et al. 2008). In classical biological control programs, the use of more specialized natural enemies often provides a greater assurance that nontarget risk is low, which is a key consideration of importation programs (Hoddle 2004; van Lenteren et al. 2006). Nevertheless, $P$. lounsburyi has not often been used in biological control programs for B. oleae, in part because of greater difficulty in rearing this species (Daane et al. 2008). Although P. lounsburyi appears to be specific to $B$. oleae, members of the $P$. concolor species complex are oligophagous, having been reared from other tephritid host species (e.g. Copeland et al. 2004; Wharton 1989).

Psyttalia concolor has been used widely for B. oleae biological control in Europe (Daane and Johnson 2010; Rugman-Jones et al. 2009), in contrast to $P$. lounsburyi and due, in part, because it has been easily reared on the Mediterranean fruit fly (Medfly), Ceratitis capitata (Weidemann) (Kapatos et al. 1977; Monastero and Delanoue 1966). The origin of $P$. concolor used in most of the early European field releases was Tunisia (Rugman-Jones et al. 2009) and, for this reason, material collected throughout much of Europe today comprises relatively similar genetic groupings of $P$. concolor (Karam et al. 2008). Psyttalia humilis (Silvestri) is a close relative of $P$. concolor, first described with material reared from Medfly collected in South Africa (Rugman-Jones et al. 2009). The two species are, morphologically, virtually indistinguishable and $P$. humilis has often been treated as a junior synonym of $P$. concolor or described as $P$. cf. concolor (cf. = color form) (Kimani-Njogu et al. 2001; Wharton et al. 2000). However, recent genetic analysis showed clear separation of the north African P. concolor and the sub-Saharan $P$. humilis (Rugman-Jones et al. 2009). Prior to this genetic separation, $P$. humilis (as $P$. cf. concolor) was 
imported to California from a insectary colony in Guatemala, provided USDA APHIS PPQ release permission, and later released in California as $P$. cf. concolor (Yokoyama et al. 2008a; Yokoyama et al. 2008b). The more recent Rugman-Jones et al. (2009) work provided molecular tools for separation of various populations and illustrated the molecular relationships among identifiable groups of Psyttalia populations. These authors suggest that $P$. humilis is an available name for sub-Saharan populations that are morphologically similar to $P$. concolor, and we have elected to use this description to provide separation of the material used in this study as compared with north African derived P. concolor individuals used in many of the earlier European studies (e.g. Biliotti and Delanoue 1959; Kapatos et al. 1977; Monastero and Delanoue 1966; Neuenschwander et al. 1983).

Well-debated risks and benefits are associated with classical biological control, and quarantine evaluations alone may not adequately describe a natural enemy's field performance (Babendreier et al. 2005; Messing and Wright 2006). For these reasons, individuals from established colonies of the imported $P$. humilis and $P$. lounsburyi were evaluated in cage trials after approval for field release had been granted from the USDA APHIS PPQ. Field cages have long been used to estimate the impact of natural enemies, where manipulated densities of both the targeted herbivore and the tested natural enemy can be tested under natural field conditions (Luck et al. 1988). Our primary goal was to determine if $P$. lounsburyi and/or $P$. humilis could parasitize $B$. oleae in cultivated olives under typical field conditions in California. Our secondary goals were to directly compare $P$. lounsburyi and $P$. humilis, and evaluate abiotic factors that might hinder their effectiveness. Comparative evaluation among $P$. lounsburyi and $P$. humilis is lacking both in laboratory and field studies. Here, we compared individuals from a $P$. lounsburyi population originally collected in Kenya, and individuals from two $P$. humilis populations (Kenya and Namibia). The $P$. humilis Kenya (Ka) colony was originally established with parasitoids collected from tephritids infesting coffee in Kenya (Wharton et al. 2000) and reared on C. capitata in artificial diet at the USDA APHIS PPQ, Moscamed biological control laboratory, San Miguel Petapa, Guatemala (Yokoyama et al. 2008a). The P. humilis Ka was previously referred to $P$. cf. concolor (Kimani-Njogu et al. 2001; Wharton et al. 2000; Yokoyama et al. 2008a) or P. concolor (Wang et al. 2009b, c). The P. humilis Namibia (Na) colony was originally established from parasitoids reared from $B$. oleae collected on wild olives in Namibia (Daane et al. 2011). The P. humilis Na was previously referred to as $P$. humilis (Rehman et al. 2009). Prior to all studies, we used molecular methods, as described by Rugman-Jones et al. (2009), to confirm the relationship of both colonies remained true to the original field-collected populations.

Cage studies were conducted in different regions and seasonal periods to permit inclusion of abiotic parameters that were previously identified as being influential on B. oleae and its parasitoids. Relative to climate, olives are grown in California's coastal regions that are characterized by mild temperatures and in interior valleys that are characterized by hot, dry summers. In the warmer interior valleys, fly population growth may be limited during periods of high summer temperature (Johnson et al. 2011), which increases adult fly mortality and reduces their movement and oviposition success (Wang et al. 2009a). This might also impact parasitoid establishment and effectiveness in California via direct mortality of Psyttalia as developing larvae or as foraging adults (Daane et al. 2008; Sime et al. 2006a; Yokoyama et al. 2010). Similarly, the failure of $P$. concolor to establish in southern Europe was partly contributed to unsuitable climatic conditions (Raspi and Loni 1994). Second, fruit size may affect parasitism rate. For example, some parasitoid species are less effective at attacking $B$. oleae larva feeding deep inside larger olive fruit because their ovipositors are too short to penetrate the fruit pulp to reach the fruit fly larva. Researchers have suggested that some of the more specialized Psyttalia species have adapted to $B$. oleae larvae feeding within smaller wild olive fruit, where the fruit fly larva are relatively close to the fruit surface and easily reached (Wang et al. 2009b, c). Third, seasonal changes may impact parasitoid survival through the availability and condition of both $B$. oleae and the olive fruit. Here, we evaluated the relative efficiency of $P$. lounsburyi and $P$. humilis in field-cage tests in two widely separated climatic zones, on differently-sized fruit cultivars, and during different seasonal periods. A better understanding of how these abiotic parameters affect field parasitism rates would provide invaluable 
information for optimal release of parasitoids in California. Results are discussed with respect to host specialization of the specialist $P$. lounsburyi as compared with the oligophagous $P$. humilis.

\section{Materials and methods}

Insect cultures

Laboratory colonies of $B$. oleae, $P$. lounsburyi, and both $\mathrm{Ka}$ and $\mathrm{Na}$ colonies of $P$. humilis were maintained on olive fruit held in a controlled room $\left(24 \pm 2^{\circ} \mathrm{C}, 16 \mathrm{~L}: 8 \mathrm{D} \mathrm{h}, 40-60 \% \mathrm{RH}\right)$ at the University of California Kearney Research and Education Center (KREC), Parlier, California, USA. Olives used were from an untreated KREC orchard in which four major California cultivars were available: Sevillano, Ascolano, Manzanillo, and Mission, in the order of decreasing fruit size (see Wang et al. 2009b). California olive cultivars have fruit maturing from late summer to late winter in California (Burrack and Zalom 2008). Some fruit of Manzanillo or Mission cultivars can stay on trees through winter and spring, and these fruit provided acceptable host material for year-round production of B. oleae cultures (Wang, unpublished data).

The B. oleae colony was established in 2003 from flies collected in Davis, California, and has been maintained on olive fruit with additions of fieldcollected flies each year to maintain colony vigor. Adult flies were held in screened cages (Bug Dorm2, BioQuip, Rancho Dominguez, CA, USA) and were provided water, honey, and hydrolyzed yeast (Fisher Biotech, Fairlawn, NJ, USA). Olives were exposed to gravid adult females until each fruit had 3-5 oviposition stings. Infested olives were then distributed over a piece of wire mesh that rested $2 \mathrm{~cm}$ above a plastic tray $(36 \times 18 \times 10 \mathrm{~cm})$. Larvae matured after 9-12 days and dropped into the tray where puparia were collected and placed in Bug Dorm2 holding cages.

The colony of $P$. lounsburyi was derived from about 200 female and 200 male wasps imported in July 2006 from the USDA-ARS European Biological Control Laboratory (EBCL) in Montferrier, France. The original EBCL colony was started from parasitized $B$. oleae collected in wild olives in the Burguret Forest, Kenya. The colony was initially maintained on B. oleae in olives, but was later reared on C. capitata cultured on artificial diet for 11-13 generations before arrival in California. At KREC, infested olives containing early third instar $B$. oleae were exposed to parasitoids in Bug Dorm2 holding cages. After a 2-3 days exposure period, the olives were transferred to plastic trays, as described above, to rear parasitized larvae and collect pupae that dropped from the fruit. Additional parasitoids shipped from EBCL were added to the colony each year to boost the KREC colonies. All parasitoids used in the described experiments were reared on B. oleae for two or more generations at KREC before each trial began. The Ka and $\mathrm{Na}$ colonies of $P$. humilis were maintained since October 2007 at KREC on B. oleae; the two colonies were reared in separate rooms, using similar methods as described for P. lounsburyi.

\section{Field sites}

Field-cages were used at one coastal and two interior valley sites. The coastal site was in San Luis Obispo (SLO), California, where the range of 30 year average winter and summer temperatures (low to high) was 5.5-17. $7^{\circ} \mathrm{C}$ (January) and $11.5-26.1^{\circ} \mathrm{C}$ (July), based on California Irrigation Management Information System (CIMIS) weather station (UCIPM 2010). The interior valley sites were at KREC and the University of California Lindcove Research and Experiment Station (LREC) in Lindcove, California, USA, where there were colder winter and warmer summer 30 year mean temperature ranges (low to high): $3.0-13.4^{\circ} \mathrm{C}$ (January) and $16.5-34.6^{\circ} \mathrm{C}$ (July) for $\mathrm{KREC}$, and 4.6-15. $8^{\circ} \mathrm{C}$ (January) and $17.9-35.2^{\circ} \mathrm{C}$ (July) for LREC (UCIPM 2010). The 30 year average annual precipitation was $63.1,31.5$, and $29.7 \mathrm{~cm} \mathrm{year}^{-1}$, for SLO, KREC, and LREC, respectively (UCIPM 2010). Most cage studies were conducted at KREC where $B$. oleae densities could be more easily manipulated. LREC was used for the spring and early summer trails in 2007, when fruit load on trees at KREC was insufficient.

\section{Experimental design}

Olive branches with $40-60$ fruit were isolated in 18.91 screened cages (45 cm long, $25 \mathrm{~cm}$ diam., Tufpro Nylon Paint Strainers, Warren County, NC, USA). Unless otherwise mentioned, all olives were Manzanillo $c v$., which is one of the more common 
olive cultivars used in California and is representative of a small olive fruit (Burrack and Zalom 2008). A piece of cardboard was placed over each cage to block direct sunlight, which was observed to affect parasitoid foraging in the cage and to increase internal temperature. A ring of Tanglefoot was placed around the branch, external to and above each cage, as a barrier for foraging ants.

For tests at interior valley sites (KREC and LREC), ten gravid female $B$. oleae were released into each cage to establish a larval infestation in the enclosed fruit. These cages were checked daily to determine the level of fruit infestation on ten randomly selected fruit. Adult flies were removed when each fruit had 3-5 oviposition marks. At the coastal site, the fruit were naturally infested with $B$. oleae larvae and no inoculation was needed. However, because infested fruit contained different B. oleae development stages, puparia were collected during the first two weeks to estimate host density. Parasitoids were released into the cages when $3 \mathrm{rd}$ instar $B$. oleae were available because this is the preferred host stage for oviposition (Daane et al. 2008; Sime et al. 2006a). Into each cage, 15 or 20 female wasps (depending on the availability of parasitoids) were released, with water and honey provided for adult parasitoid feeding. After a two week exposure period, the cages were removed, the number of live parasitoids (F1) recorded, and the fruit were taken to the laboratory for emergence of parasitoid offspring (F2) and flies. This exposure time reflected the estimated developmental time of $3 \mathrm{rd}$ instar $B$. oleae to pupae and assured that none of the F2 parasitoids emerged inside the cages.

In the laboratory, collected fruit were placed in plastic containers $(11 \times 11 \mathrm{~cm})$ covered with organdy cloth and fitted with a raised metal grid ( $2 \mathrm{~cm}$ high) on the bottom, which suppressed mold formation and allowed pre-pupal flies to drop from the fruit and through the grid to the bottom of the container, where they could be easily collected. For each test, olive fruit were dissected and dead or unemerged puparia were reconstituted in water for one day and then dissected under a microscope to determine the presence or absence of recognizable immature parasitoid cadavers and pharate adults. Parasitism was estimated based on the number of emerged and dissected wasps and flies, while host density was estimated based on the total fly puparia. At the end of trial, the number of surviving wasps was recorded. Percentage parasitism may not be the best comparative measurement of field performance in these trials because of variations in trial location and season, as well as variation in the host density during the two week exposure period. For this reason, the number of offspring produced per female per cage was also used for comparisons.

This design was used in each of 24 trials, with ten replicates (cages) per trial, conducted from 2006 to 2009. Key parameters that varied among trials were: season, site location, parasitoid species and/or population, and host density in each cage. Most trials were conducted at KREC, where B. oleae densities could be best manipulated, and between September and December, when $B$. oleae would naturally be available in mature fruit. Included in the 24 trials were six paired trials, conducted to compare colonies of $P$. humilis Ka or Na versus $P$. lounsburyi. For each of these paired trials, only one tree was used to avoid possible variation of fruit size and maturity, as well as microhabitat conditions among different trees. Cages bearing infested olive fruit were randomly assigned to each parasitoid species, and all other test conditions (i.e., number of wasps per cage and exposure time) were the same for both parasitoid species. Parasitism levels in cultivars with large fruit (Ascolano $c v$.) versus small fruit (Manzanillo $c v$.) were also compared in two of the six paired trials (4 October 2007). In these trials, fruit size was further controlled by sorting and removing extremely large or small fruit from each cultivar.

\section{Data analysis}

Data are presented as means $( \pm \mathrm{SE})$ and were derived from trial means rather than individual cages. Climatic data were obtained from the nearest CIMIS station. For comparison of field survival of adult parasitoids (F1), offspring production (F2), and the impact of season, climate, and region, we pooled data from all cage trials, with the exception of the trial using the Ascolano cultivar. Data were arcsine square-root transformed, when needed, to satisfy the assumptions of ANOVA. A multiple regression model was employed to analyze the effects of parasitoid species, ratio of hosts to parasitoids, and climatic factors (mean field temperature and $\mathrm{RH}$ ) on parasitism levels. For this analysis, the parasitoid species was treated as a category variable, and coded as 0 for $P$. lounsburyi and 1 for $P$. humilis. For paired comparisons, a one-way 
ANOVA was performed. All analyses used JMP software (V. 8, SAS 2008, Cary, NC, USA).

\section{Results}

A total of 24 trials were completed (240 cages), with 2, 7, 12, and 3 trials in 2006, 2007, 2008, and 2009 , respectively. Ten trials were conducted with $P$. lounsburyi, nine with $P$. humilis $\mathrm{Na}$, and five with $P$. humilis Ka. Most trials were conducted at KREC (14), followed by San Luis Obispo (six) and LREC (four). Trials were conduced in the fall season to coincide with the natural availability of $B$. oleae in mature fruit $(1,11$, and 1 trials in late September, October, and November, respectively), in late-winter to spring to coincide with emergence of $B$. oleae and parasitoids from overwintered fruit (1,3, and 1 trials in February, March and April, respectively), and during the summer on green and maturing fruit that would sustain B. oleae (1, 2, 2, and 1 trials in May, June, August, and early September, respectively).

\section{Parasitoid performance}

Psyttalia lounsburyi and P. humilis successfully attacked B. oleae at all locations and seasonal periods, averaging $32.4 \pm 4.1 \%$ parasitism across all trials and ranging from $1.0 \pm 1.0 \%$ (KREC, 5 September 2006) to $86.4 \pm 2.2 \%$ (KREC, 12 November 2008). Across all trials, percentage parasitism by $P$. humilis (Ka and Na) was higher than that by $P$. lounsburyi $\left(\mathrm{F}_{2,21}=\right.$ 15.156, $P<0.001$ ) (Fig. 1a).

Host density averaged $78.2 \pm 7.5$ B. oleae larvae per cage, ranging from $12.1 \pm 1.3$ (LREC, 15 July 2007 ) to $154.6 \pm 29.4$ larvae per cage (SLO, 7 October 2008). Based on the 15 or 20 adult female parasitoids released per cage, the initial host:parasitoid ratio averaged $5.4 \pm 0.5$, and ranged from $0.8 \pm 0.1$ and $13.2 \pm 1.7$ ratios of host:parasitoid per cage. Host density did not, however, differ among parasitoid treatments $\left(\mathrm{F}_{2,21}=1.045, P=0.369\right)$, and in all trials more $B$. oleae than parasitoids emerged, suggesting sufficient host material was available. Therefore, the number of offspring produced per female was used as an additional comparative measurement of parasitoid performance. Across all trials, more offspring were produced by $P$. humilis Na than $P$. lounsburyi $\left(\mathrm{F}_{2,21}=7.135, P=0.004\right)$

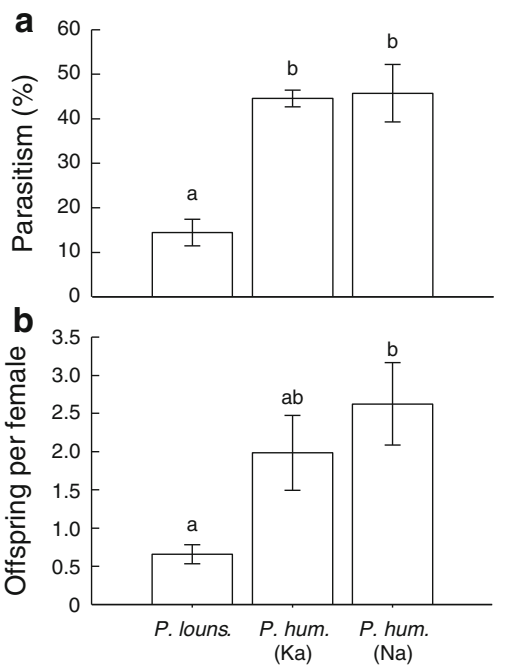

Fig. 1 a Percentage parasitism $( \pm \mathrm{SE})$ for $P$. lounsburyi (P. louns.) and P. humilis (P. hum.) populations from Kenyan (Ka) and Namibia (Na) following a two week exposure period in cage trials. b Offspring produced per female (mean $\pm \mathrm{SE}$ ) following a two week exposure period for $P$. lounsburyi, $P$. humilis $\mathrm{Ka}$ and $P$. humilis $\mathrm{Na}$. In both graphs, data are combined from cage trials conducted in California's interior valley and coastal areas, and across fall, spring, and summer seasonal periods. Different letters above each bar indicate a significant difference between mean values (Tukey's HSD, $P<0.05)$

(Fig. 1b). Similarly, for each of the six comparative tests, parasitism by $P$. humilis (both $\mathrm{Ka}$ and $\mathrm{Na}$ colonies) as indicated by offspring per female was higher than $P$. lounsburyi regardless of fruit size, location, or trial date (Fig. 2a).

Abiotic factors

No difference was found in the production of offspring per cage (mean \pm SE) between the interior valley $(32.0 \pm 4.9)$ and coastal $(33.7 \pm 8.1)$ locations $\left(\mathrm{F}_{2,21}=0.028, P=0.867\right)$. A seasonal effect was observed, with more offspring per cage produced in spring than in summer $\left(\mathrm{F}_{2,21}=3.457, P=0.050\right)$ (Fig. 3a). The seasonal effect was directly related to temperature, which averaged $14.7 \pm 1.5,15.3 \pm 0.6$, $22.5 \pm 1.4^{\circ} \mathrm{C}$ in spring, fall, and summer trials, respectively $\left(\mathrm{F}_{2,21}=15.92, P<0.001\right)$.

Multiple regression analyses showed that parasitism decreased with increased field temperature (Table 1). The significantly positive estimate value for the dummy variable (parasitoid species) indicated that parasitism was overall higher by $P$. humilis than 

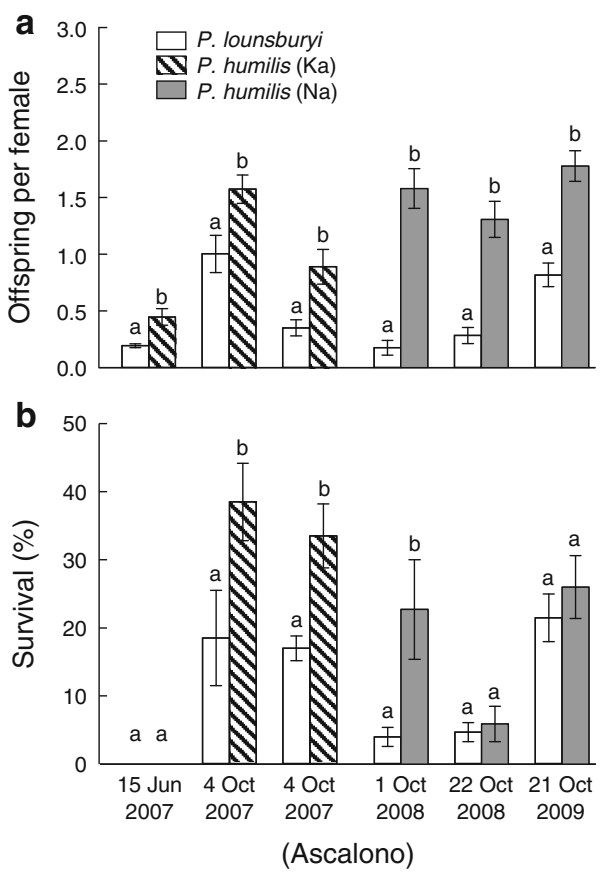

Fig. 2 Treatment results (mean \pm SE) from six paired cage trials for $\mathbf{a}$ offspring produced per female and $\mathbf{b}$ percentage survival of adult parasitoids during the two week exposure period for $P$. lounsburyi versus $P$. humilis Ka (2007 trials) and $P$. lounsburyi versus $P$. humilis $\mathrm{Na}$ (2008 and 2009 trials). All trials were conducted with fruit of Manzanillo cv. with the exception of the second 4 October 2007 trial, which was conducted with fruit of Ascolano $c v$. In each graph, different letters above each bar indicate a significant difference between mean values (Tukey's HSD, $P<0.05$ )

P. lounsburyi. The ratio of host to parasitoid had no significant effect on the parasitism. However, mean relative humidity ( $\mathrm{RH}$ ) was negatively related to mean field temperature $\left(T^{\circ} \mathrm{C}\right)$ (linear regression slope: $\left.T\left({ }^{\circ} \mathrm{C}\right)=29.3-0.21(\mathrm{RH}), F_{2,23}=38.7, P<0.001\right)$, indicating no rain events or significant morning dew occurred in the trial periods during the dry summer months, for the interior valley locations.

Across all trials, adult parasitoid (F1) survival rate during the exposure period was $11.9 \pm 4.4,22.5 \pm$ 6.8 and $28.0 \pm 13.3 \%$ for $P$. lounsburyi, $P$. humilis $\mathrm{Ka}$, and $P$. humilis $\mathrm{Na}$, respectively. As with offspring production, linear regression analyses showed percentage survival of parasitoids was negatively correlated with mean field temperatures during the exposure period (Table 2), and the data were fit to a two parameter, inverse polynomial model to graphical show the relationship between temperature and adult parasitoid survival (Fig. 3b).
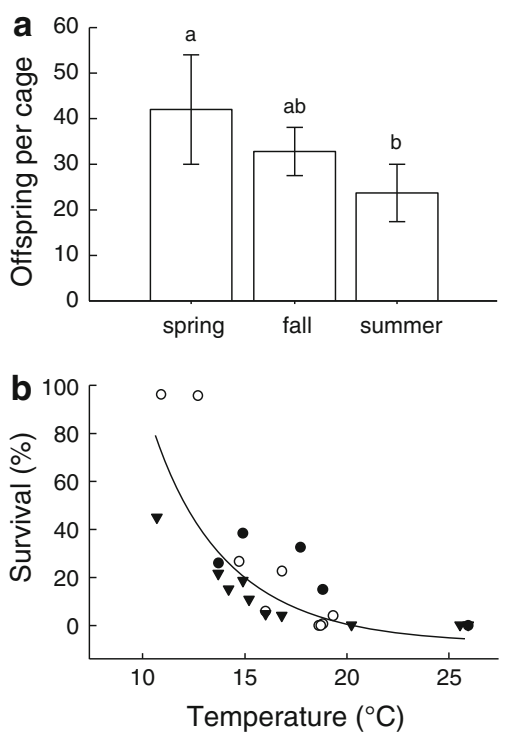

Fig. 3 Effect of seasonal variation in temperature during field cage trials on the a offspring per cage (mean \pm SE) for trials conducted in summer, fall, and spring periods and $\mathbf{b}$ the relationship between percentage adult survival and mean temperature during the two week exposure period for individual cage trials. For analysis, data were combined for all parasitoid species and populations from all cage trials. In the bar graph, different letters above each bar indicate a significant difference between mean values (Tukey's HSD, $P<0.05$ ). In the regression graph, different symbols indicate data for $P$. lounsburyi (inverted filled triangle), $P$. humilis $\mathrm{Ka}$ (filled circle), and $P$. humilis $\mathrm{Na}$ (open circle); the data were fit to a two parameter, inverse polynomial model ( $\mathrm{f}=\mathrm{y}_{0}+$ $\left.(a / \mathrm{x})+\left(b / \mathrm{x}^{2}\right)\right)$ to graphical show the relationship between temperature and adult parasitoid survival, where $\mathrm{y}_{0}=18.9$, $a=-1374.8, b=21,326 ; \mathrm{F}_{2,21}=17.45 ; P<0.001$

Table 1 Parameters of multiple regression analyses show significant $\left(F_{4,19}=10.9, P<0.001\right)$ effects of parasitoid species, ratio of hosts to parasitoids, and climate factors on resultant patterns of parasitism of larval B. oleae in field-cage tests on olive cultivar of Manzanillo in California

\begin{tabular}{lcrr}
\hline Parameters & Estimate $( \pm \mathrm{SE})$ & \multicolumn{1}{l}{ t } & P-value $^{a}$ \\
\hline Intercept & $98.93 \pm 33.49$ & 2.95 & 0.008 \\
Parasitoid species $^{\mathrm{a}}$ & $32.09 \pm 5.17$ & 6.21 & $<0.001$ \\
Raito of hosts & $-0.44 \pm 0.97$ & -0.45 & 0.657 \\
$\quad$ to parasitoids & & & \\
Mean field temperature & $-2.44 \pm 1.05$ & -2.33 & 0.031 \\
Mean RH\% & $-0.71 \pm 0.28$ & -2.56 & 0.019 \\
\hline
\end{tabular}

a For categorical variables parasitoid species was coded as ' 0 ' for $P$. lounsburyi and ' 1 ' for $P$. humilis 
Table 2 Relationship between mean field temperature $(X)$ and percentage survival $(Y)$ of two larval $B$. oleae parasitoids at the end of exposure in field-cage tests in California

\begin{tabular}{lllllr}
\hline Parasitoids $^{\mathrm{a}}$ & Relationship & $R^{2}$ & DF & \multicolumn{1}{l}{$P$} & \multicolumn{1}{l}{$P$} \\
\hline P. humilis (Ka) & $Y=72.1-0.69 X$ & 0.7097 & 1,5 & 9.78 & 0.026 \\
P. humilis (Na) & $Y=227.1-12.3 X$ & 0.8576 & 1,8 & 42.16 & $<0.001$ \\
P. lounsburyi & $Y=49.4-2.2 X$ & 0.6313 & 1,10 & 15.41 & 0.003 \\
\hline
\end{tabular}

${ }^{a} \mathrm{Ka}$ Kenyan population, $\mathrm{Na}$ Namibian population

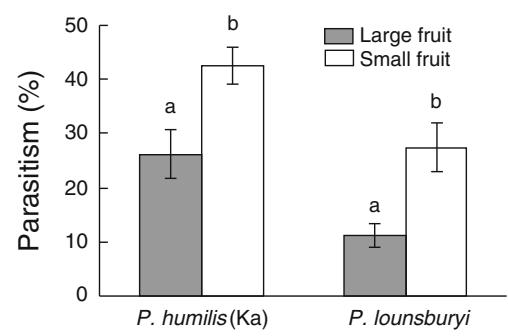

Fig. 4 Percentage parasitism $( \pm \mathrm{SE})$ by $P$. lounsburyi and $P$. humilis $\mathrm{Ka}$ on $B$. oleae larvae feeding in the small (fruit typical of Manzanillo $c v$.) and large (fruit typical of Ascolano $c v$.) fruit. Different letters above each bar indicate a significant difference between mean values (Tukey's HSD, $P<0.05$ )

In the six paired trials, no parasitoids were found alive for the first trial (LREC, 15 June 2007) when temperatures averaged $25.8 \pm 1.8^{\circ} \mathrm{C}$. For all other paired trials, percentage survival of $P$. humilis (both $\mathrm{Ka}$ and $\mathrm{Na}$ colonies) was either significantly higher than $P$. lounsburyi or there was no significant difference between both species (Fig. 2b). Trial temperatures (mean $\pm \mathrm{SE}$ (range)) during the exposure period were as follows: 15 June 2007: $25.8 \pm$ $0.5^{\circ} \mathrm{C}\left(15.4-35.4^{\circ} \mathrm{C}\right), 4$ October $2007: 14.8 \pm 0.6^{\circ} \mathrm{C}$ $\left(6.7-23.5^{\circ} \mathrm{C}\right), 1$ October 2008: $16.7 \pm 1.1^{\circ} \mathrm{C}(8.3-$ $\left.26.2^{\circ} \mathrm{C}\right), 22$ October 2008: $15.9 \pm 0.9^{\circ} \mathrm{C}(10.0-$ $25.1^{\circ} \mathrm{C}$ ), and 20 October 2009: $13.6 \pm 0.8^{\circ} \mathrm{C}$ $\left(6.3-22.7^{\circ} \mathrm{C}\right)$. Parasitism of $B$. oleae was significantly higher in the smaller Manzanillo $c v$. fruit than in the larger Ascolano $c v$. fruit for both species ( $P$. humilis Ka: $F_{1,19}=10.9, P<0.05 ; P$. lounsburyi: $F_{1,19}=$ 15.4, $P<0.001$ ) (Fig. 4).

\section{Discussion}

We demonstrated that $P$. lounsburyi and $P$. humilis can successfully reproduce on $B$. oleae in California olives under varying environmental conditions. Of the two parasitoid species tested, $P$. humilis was more effective than $P$. lounsburyi, regardless of season, olive growing region, or fruit cultivars. The difference between these closely related Psyttalia species may have resulted from differences in their ovipositor lengths relative to the fruit size of commercial olives. In paired tests, fruit size affected parasitism levels by both $P$. lounsburyi and $P$. humilis, with higher rates of parasitism for $B$. oleae in the smaller Manzanillo $c v$. than the larger Ascolano $c v$. fruit. This was consistent with laboratory studies in which Wang et al. (2009b) and Daane et al. (2008) suggest that the relatively short ovipositor lengths of $P$. humilis (reported as $P$. concolor) and $P$. lounsburyi, respectively, might be an impairment for their successful oviposition when $B$. oleae feeds in larger cultivated fruit compared with smaller wild fruit. In a companion study, the ovipositor length of $P$. lounsburyi was reported as significantly shorter than that of P. humilis (Wang et al. 2009c), which may further reduce the parasitism rates by $P$. lounsburyi relative to $P$. humilis. Wang et al. (2009c) hypothesize that olive domestication, which resulted in larger fruit, created a structural refuge for larval $B$. oleae from the attack by more specialized braconid parasitoids that evolved in association with B. oleae in the smaller, wild olive fruit. The influence of a parasitoid's ovipositor length and parasitoid effectiveness relative to fruit fly feeding habits and fruit morphology has similarly been discussed by other researchers (Sime et al. 2007; Sivinski et al. 2001).

Specialization is thought to confer superior hostlocation efficiency and greater ability to overcome host defenses such that a host specialist should locate and parasitize more hosts than a generalist in a given arena (e.g. Wang and Keller 2002). However, in its presumably native regions, $P$. lounsburyi was relatively rare in recent surveys in South African (Mkize et al. 2008) and Namibia (Daane et al. 2011), but was a dominant parasitoid in Kenya (Copeland et al. 2004). The results may depend on whether researchers were collecting from wild or cultivated olives because, as discussed previously, of the interaction between fruit size and ovipositor length. The discrepancy may also be due to limitation of $P$. lounsburyi related to environmental tolerances. We note here that the original $P$. lounsburyi used in this study was from a population collected in Kenya at a location near the equator and at about $1500 \mathrm{~m}$ in elevation, providing year-round mild climate and 
wild olive fruit. Other $P$. lounsburyi populations, such as those in South Africa, may be better suited for use in classical biological control in olive growing regions with hot, dry summer climates. Such regional differences in parasitoid populations have yet to be fully accounted for in the survey and importation of B. oleae natural enemies.

Classical biological control, to be successful, may involve a significant change in both host habitat and climatic conditions. In this particular case, it can also involve a modification of the tri-tropic interaction as crop domestication has modified natural hostparasitoid interactions, which may not favor more specialized parasitoids. The fact that the oligophagous $P$. humilis performed better in field cage trials suggests that host and climatic adaptability may be a necessary trait for B. oleae control in California. In fact, during the recent foreign exploration surveys and collections, only on Mt. Kenya was $P$. lounsburyi the dominant $B$. oleae parasitoid, $U$. africana was the dominant parasitoid in South Africa (Daane et al. 2011), but was not successfully reared in quarantine on $B$. oleae in cultivated olive fruit. In quarantine trials, $D$. kraussii and $D$. longicaudata, two polyphagous fruit fly parasitoids that have relatively long ovipositors, were easily reared on B. oleae (Sime et al. 2006b) but have not been released due to concerns of non-target impacts. However, these Dichasmimorpha species, and other species with a relatively wide host range, may have to be reconsidered if biological control of $B$. oleae is a primary need (Wang et al. 2009b).

Adult survival during periods of high summer temperatures was another factor that may have lowered $P$. lounsburyi effectiveness relative to $P$. humilis in our studies. Results from paired cage trials and multiple factors analyses showed that both field temperature and humidity affected the patterns of parasitism. In laboratory studies, Daane et al. (2008) also suggested that, P. lounsburyi had a lower upper threshold for development and poorer adult survival at higher temperatures relative to a laboratory colony of $P$. concolor tested under similar conditions (Sime et al. 2006a). The results of the current field cage study and the previous laboratory study suggest $P$. lounsburyi may not permanently establish in California interior valleys unless cooler microclimates within the orchard can provide refuge from high summer temperatures. Climate matching of imported $B$. oleae parasitoid species or subspecies to release sites has rarely been emphasized, despite its obvious importance (Neuenschwander 1982). Even different laboratory cultures of $P$. concolor with different rearing histories differed in tolerance to temperature extremes (Sime et al. 2006a). Nevertheless, there may be a role for these natural enemies in California's coastal regions, where high $B$. oleae populations may more naturally occur (Burrack et al. 2008; Yokoyama et al. 2006) and where smaller fruit on olive oil cultivars are more common. In contrast, biological controls are less likely to be successful for table olives in California's interior valleys, where a number of factors may reduce parasitoid impact: (1) table olive cultivars and management practices result in larger olive fruit size, (2) there is a lower tolerance for infested fruit marketed for table olives, and (3) higher summer temperatures may limit both fly and natural enemy populations (Daane et al. 2008; Loni 1997; Wang et al. 2009a).

Although the efficiency of both the tested larval parasitoid species on B. oleae is limited on large fruit cultivars, they can still be effective biological control agents on small fruit cultivars. Additionally, landscape olives (e.g. urban areas, or abandoned trees, and other non-crop habitats) often have smaller fruit because of the lack of irrigation and management. These trees may support substantial $B$. oleae populations and provide reservoirs of the pest where insecticidal sprays cannot or are rarely used. The introduced parasitoids would show greater promise for biological control in such landscape olives, thereby reducing the inoculum source of the pest for commercial olives. Also, small olive fruit (e.g. Mission $c v$. and Manzanillo $c v$.) are predominantly grown for olive oil, and oil olives can withstand low levels of infestation (Johnson et al. 2006). Release of either $P$. humilis or P. lounsburyi may have increased impact under these situations as compared with commercial table olives. Factors that could also affect future parasitoid establishment on B. oleae in California include the availability of host flies through the year, parasitoid overwintering success, parasitoid searching efficiency at low host densities, and parasitoid survival mechanisms during periods when properly sized $B$. oleae or olive fruit are unavailable.

Acknowledgments We are grateful to Martha Gerik (University of California, Riverside) for assistance in all field 
tests; David Headrick, Pete Peterson and Therese Kapaun (California Polytechnic State University, San Luis Obispo) for facilitating field studies in San Luis Obispo; Walt French, Scott Ritterbuck, Anne May and David Righetti for allowing us to use their olive trees in San Luis Obispo; Arnaud Blanchet and Walker Jones (USDA ARS European Biological Control Laboratory, Montferrier, France) and Pedro Rendón (USDAAPHID-PPQ, Moscamed biological control laboratory, San Miguel Petapa, Guatemala) for providing the initial colonies of parasitoids; John Andrews (University of California, Berkeley) for managing the quarantine importation; and Mathew Middleton (University of California, Berkeley) for molecular analysis of parasitoid colonies. Funds were provided by the California Specialty Crop Block Grant, California Olive Committee, USDA APHIS and CDFA Biological Control Program, and USDA CSREES Special Grants Program: Pest Management Alternatives to MWJ and KMD.

Open Access This article is distributed under the terms of the Creative Commons Attribution Noncommercial License which permits any noncommercial use, distribution, and reproduction in any medium, provided the original author(s) and source are credited.

\section{References}

Augustinos AA, Stratikopoulos EE, Zacharopoulou A, Mathiopoulos KD (2002) Polymorphic microsatellite markers in the olive fly, Bactrocera oleae. Mol Ecol Notes 2:278-280

Babendreier D, Bigler F, Kuhlmann U (2005) Methods used to assess non-target effects of invertebrate biological control agents of arthropod pests. BioControl 50:821-870

Biliotti E, Delanoue P (1959) Contribution à l'étude biologique d'Opius concolor Szepl. (Hym.: Braconidae) en élevage de laboratoire. Entomophaga 4:7-14

Burrack HJ, Zalom FG (2008) Olive fruit fly (Diptera: Tephritidae) ovipositional preference and larval performance in several commercially important olive varieties in California. J Econ Entomol 101:750-758

Burrack HJ, Connell JH, Zalom FG (2008) Comparison of olive fruit fly (Bactrocera oleae (Gmelin)) (Diptera: Tephritidae) captures in several commercial traps in California. Int J Pest Manage 54:227-234

Collier TR, van Steenwyk RA (2003) Prospects for integrated control of olive fruit fly are promising in California. Calif Agric 57:28-31

Copeland RS, White IM, Okumu M, Machera P, Wharton RA (2004) Insects associated with fruits of the Oleaceae (Asteridae, Lamiales) in Kenya, with special reference to the Tephritidae (Diptera). Bishop Mus Bull Entomol 12:135-164

Daane KM, Johnson MW (2010) Olive fruit fly: managing an ancient pest in modern times. Annu Rev Entomol 55:155-169

Daane KM, Sime KR, Wang X-G, Nadel H, Johnson MW, Walton VM (2008) Psyttalia lounsburyi (Hymenoptera: Braconidae), potential biological control agent for the olive fruit fly in California. Biol Control 44:78-89
Daane KM, Johnson MW, Pickett CH, Sime KR, Wang X-G, Nadel H, Andrews JW, Hoelmer KA (2011) Biological control of the olive fruit fly in California. Calif Agric (in press)

Hoddle MS (2004) Restoring balance: using exotic species to control invasive exotic species. Conserv Biol 18:38-49

Johnson MW, Zalom FG, Van Steenwyk R, Vossen P, Devarenne AK, Daane KM, Krueger WH, Connell JH, Yokoyama V, Bisabri B, Caprile J, Nelson J (2006) Olive fruit fly management guidelines for 2006. Univ Calif Plant Prot Quart 16:1-7

Johnson MW, Wang X-G, Nadel H, Opp SB, Lynn-Patterson K, Stewart-Leslie J, Daane KM (2011) High temperature impacts olive fruit fly population dynamics in California's Central Valley. Calif Agric (in press)

Kapatos E, Fletcher BS, Pappas S, Laudeho Y (1977) Release of Opius concolor and Opius concolor var siculus (Hym.: Braconidae) against spring generation of Dacus oleae (Dipt.: Trypetidae) on Corfu. Entomophaga 22:265-270

Kapaun T, Nadel H, Headrick D, Vredevoe L (2010) Biology and parasitism rates of Pteromalus nr. myopitae (Hymenoptera: Pteromalidae), a newly discovered parasitoid of olive fruit fly Bactrocera oleae (Diptera: Tephritidae) in coastal California. Biol Control 53:76-85

Karam N, Guglielmino CR, Bertin S, Gomulski LM, Bonomi A, Baldacchino F, Simeone V, Malacrida AR (2008) RAPD analysis in the parasitoid wasp Psyttalia concolor reveals Mediterranean population structure and provides SCAR markers. Biol Control 47:22-27

Kimani-Njogu SW, Trostle MK, Wharton RA, Woolley JB, Raspi A (2001) Biosystematics of the Psyttalia concolor species complex (Hymenoptera: Braconidae: Opiinae): the identity of populations attacking Ceratitis capitata (Diptera: Tephritidae) in coffee in Kenya. Biol Control 20:167-174

Loni A (1997) Developmental rate of Opius concolor (Hym.: Braconidae) at various constant temperatures. Entomophaga 42:359-366

Luck RF, Shepard BM, Kenmore PE (1988) Experimental methods for evaluating arthropod natural enemies. Annu Rev Entomol 33:367-391

Messing RH, Wright MG (2006) Biological control of invasive species: solution or pollution? Front Ecol Environ 4:132-140

Mkize N, Hoelmer KA, Villet MH (2008) A survey of fruitfeeding insects and their parasitoids occurring on wild olives, Olea europaea ssp cuspidata, in the Eastern Cape of South Africa. Biocontrol Sci Technol 18:991-1004

Monastero R, Delanoue P (1966) Lutte bioloique expérimentale contre la mouche de l'olive (Dacus oleae Gmel.) au moyen d'Opius concolor Szepl. siculus Mon. dans les îles eoliennes Sicile en 1965. Entomophaga 11:411-432

Nadel H, Daane KM, Hoelmer KA, Pickett CH, Johnson MW (2009) Non-target host risk assessment of the idiobiont parasitoid, Bracon celer (Hymenoptera: Braconidae), for biological control of olive fruit fly in California. Biocontrol Sci Technol 19:701-715

Nardi F, Carapelli A, Dallai R, Roderick GK, Frati F (2005) Population structure and colonization history of the olive fly, Bactrocera oleae (Diptera, Tephritidae). Mol Ecol 14:2729-2738 
Neuenschwander P (1982) Searching parasitoids of Dacus oleae (Gmel) (Dipt., Tephritidae) in South Africa. J Appl Entomol 94:509-522

Neuenschwander P, Bigler F, Delucchi V, Michelakis SE (1983) Natural enemies of preimaginal stages of Dacus oleae Gmel. (Dipt., Tephritidae) in Western Crete. I. Bionomics and phenologies. Bollettino del Laboratorio di Entomologia Agraria Filippo Silvestri 40:3-32

Orsini MM, Daane KM, Sime KR, Nelson EH (2007) Mortality of olive fruit fly pupae in California. Biocontrol Sci Technol 17:797-807

Raspi A, Loni A (1994) Alcune note sull'allevamento massale di Opius concolor Szepligeti (Hym.: Braconidae) e su recnti tentative d'introduzione della specie in Toscana e Liguria. Frustula Entomologia 30:135-145

Rehman JU, Wang X-G, Johnson MW, Daane KM, Jilani G, Khan MA, Zalom FG (2009) Effects of Peganum harmala (Zygophyllaceae) seed extract on the olive fruit fly (Diptera: Tephritidae) and its larval parasitoid Psyttalia concolor (Hymenoptera: Braconidae). J Econ Entomol 102:2233-2240

Rice R, Phillips P, Stewart-Leslie J, Sibbett G (2003) Olive fruit fly populations measured in central and southern California. Calif Agric 57:122-127

Rugman-Jones PF, Wharton R, van Noort T, Stouthamer R (2009) Molecular differentiation of the Psyttalia concolor (Szepligeti) species complex (Hymenoptera: Braconidae) associated with olive fly, Bactrocera oleae (Rossi) (Diptera: Tephritidae), in Africa. Biol Control 49:17-26

Silvestri F (1914) Report on an expedition to Africa in search of natural enemies of fruit flies (Trupaneidae) with descriptions, observations and biological notes, vol 3 . Hawaii Board of Agriculture and Forestry, Division of Entomology Bulletin, pp 1-146

Sime KR, Daane KM, Messing RH, Johnson MW (2006a) Comparison of two laboratory cultures of Psyttalia concolor (Hymenoptera: Braconidae), as a parasitoid of the olive fruit fly. Biol Control 39:248-255

Sime KR, Daane KM, Nadel H, Funk CS, Messing RH, Andrews JW, Johnson MW, Pickett CH (2006b) Diachasmimorpha longicaudata and D. kraussii (Hymenoptera: Braconidae), potential parasitoids of the olive fruit fly. Biocontrol Sci Technol 16:169-179

Sime KR, Daane KM, Kirk A, Andrews JW, Johnson MW, Messing RH (2007) Psyttalia ponerophaga (Hymenoptera: Braconidae) as a potential biological control agent of olive fruit fly Bactrocera oleae (Diptera: Tephritidae) in California. Bull Entomol Res 97:233-242

Sime KR, Daane KM, Wang X-G, Johnson MW, Messing RH (2008) Evaluation of Fopius arisanus as a biological control agent for the olive fruit fly in California. Agric For Entomol 10:423-431

Sivinski J, Vulinec K, Aluja M (2001) Ovipositor length in a guild of parasitoids (Hymenoptera: Braconidae) attacking Anastrepha spp. fruit flies (Diptera: Tephritidae) in southern Mexico. Ann Entomol Soc Am 94:886-895
UCIPM (2010) University of California Statewide Integrated Pest Management Program (UCIPM). http://www.ipm. ucdavis.edu/. Accessed 13 June 2010

van Lenteren JC, Bale J, Bigler E, Hokkanen HMT, Loomans AM (2006) Assessing risks of releasing exotic biological control agents of arthropod pests. Annu Rev Entomol 51:609-634

Wang X-G, Keller MA (2002) A comparison of host-searching efficiency of the two larval parasitoids of Plutella xylostella. Ecol Entomol 27:105-114

Wang X-G, Johnson MW, Daane KM, Opp S (2009a) Combined effects of heat stress and food supply on flight performance of the olive fruit fly (Diptera: Tephritidae). Ann Entomol Soc Am 102:727-734

Wang X-G, Johnson MW, Daane KM, Yokoyama VY (2009b) Larger olive fruit size reduces the efficiency of Psyttalia concolor, as a parasitoid of the olive fruit fly. Biol Control 49:45-51

Wang X-G, Nadel H, Johnson MW, Daane KM, Hoelmer K, Walton VM, Pickett CH, Sime KR (2009c) Crop domestication relaxes both top-down and bottom-up effects on a specialist herbivore. Basic Appl Ecol 10:216-227

Wharton RA (1989) Classical biological control of fruitinfesting Tephritidae. In: Robinson AS, Hooper G (eds) Fruit flies: Their Biology, Natural Enemies and Control. Elsevier, Amsterdam, pp 303-313

Wharton RA, Trostle MK, Messing RH, Copeland RS, KimaniNjogu SW, Lux S, Overholt WA, Mohamed S, Sivinski J (2000) Parasitoids of medfly, Ceratitis capitata, and related tephritids in Kenyan coffee: a predominantly koinobiont assemblage. Bull Entomol Res 90:517-526

Yokoyama VY, Miller GT, Stewart-Leslie J, Rice RE, Phillips PA (2006) Olive fruit fly (Diptera: Tephritidae) populations in relation to region, trap type, season, and availability of fruit. J Econ Entomol 99:2072-2079

Yokoyama VY, Rendon PA, Sivinsk J (2008a) Psyttalia cf. concolor (Hymenoptera: Braconidae) for biological control of olive fruit fly (Diptera: Tephritidae) in California. Environ Entomol 37:764-773

Yokoyama VY, Rendon PA, Sivinski J (2008b) Biological control of olive fruit fly (Diptera: Tephritidae) by releases of Psyttalia cf. concolor (Hymenoptera: Braconidae) in California, parasitoid longevity in presence of the host, and host status of walnut husk fly. In: Proceedings of the 7 th international symposium on fruit flies of economic importance, 10-15 September 2006. Salvador, Bahia, Brazil, pp 157-164

Yokoyama VY, Cáceres CE, Kuenen LPS, Wang X-G, Rendón PA, Johnson MW, Daane KM (2010) Field performance and fitness of an olive fruit fly parasitoid, Psyttalia humilis (Hymenoptera: Braconidae), mass reared on irradiated Medfly. Biol Control 54:90-99

Zalom FG, Van Steenwyk RA, Burrack HJ, Johnson MW (2009) Pest notes: olive fruit fly. University of California Agriculture and Natural Resources Publication 7411

Zygouridis NE, Augustinos AA, Zalom FG, Mathiopoulos KD (2009) Analysis of olive fly invasion in California based on microsatellite markers. Heredity 102:402-412 